\title{
Value of Contrast-Enhanced Ultrasound in the Differential Diagnosis of Focal Splenic Lesions
}

This article was published in the following Dove Press journal: Cancer Management and Research

\author{
Rui Yang ${ }^{1,2}$ \\ Qiang Lu' \\ Jinshun $\mathrm{Xu} \mathbb{D}^{1,2}$ \\ Jiayan Huang ${ }^{\prime}$ \\ Binyang Gao ${ }^{1,2}$ \\ Huan Zhang ${ }^{1,2}$ \\ Jie Zhou ${ }^{1,2}$ \\ Lanxin Du' \\ Feng Yan (iD) ${ }^{2}$
}

'Ultrasound Department, West China Hospital of Sichuan University, Chengdu, Sichuan, People's Republic of China; ${ }^{2}$ Laboratory of Ultrasound Imaging, West China Hospital of Sichuan University, Chengdu, Sichuan, People's Republic of China
Correspondence: Feng Yan

Laboratory of Ultrasound Imaging, West China Hospital of Sichuan University, No. 88 Keyuan Road South, Chengdu, Sichuan, 61004I, People's Republic of China

Tel +86 I8980606323

Email yan_feng@scu.edu.cn
Purpose: To identify and validate contrast-enhanced ultrasound (CEUS) features for differentiating malignant from benign splenic lesions.

Patients and Methods: Splenic lesions in 123 patients who underwent conventional ultrasound (B-mode US) and CEUS were included in this study. Two radiologists evaluated the sonograms of B-mode and CEUS. Statistical analysis was performed to identify significant imaging predictors for splenic malignant lesions. Two other radiologists independently reviewed B-mode and CEUS sonograms and diagnosed the lesions based on proposed criteria as 1) benign, 2) probably benign, 3) probably malignant or 4) malignant. The diagnostic efficiency between B-mode US and CEUS was compared.

Results: Common imaging findings of malignant lesions included hypoechoic, ill-defined margin, absence of cystic/necrotic portion, presence of splenomegaly on B-mode US, and hypoenhancement, rapid washout and presence of intralesional vessels on CEUS $(P<0.05)$. Among them, three independent features were identified using multivariate logistic regression analysis: hypoechoic pattern, hypoenhancement pattern and intralesional vessels. When three of these findings were combined as a predictor for splenic malignant lesions, 22 $(55.0 \%)$ of 40 malignant splenic lesions were identified with a specificity of $100 \%$. The diagnostic performance of two readers using receiver operating characteristic curve analysis was 0.622 and 0.533 , respectively, for B-mode US, which was significantly improved to 0.908 and 0.906 for CEUS $(P<0.001)$. The degree of other diagnostic efficiency and interreader agreement also increased with CEUS compared to B-mode US.

Conclusion: CEUS may provide more useful information than B-mode US and improve the diagnosis efficiency for distinguishing malignant from benign splenic lesions.

Keywords: spleen, splenic diseases, contrast-enhanced ultrasound, conventional ultrasound

\section{Introduction}

Focal splenic lesions (FSLs) are uncommon in comparison with those of other abdominal parenchymal organs and are often discovered incidentally. However, the differential diagnosis of FSLs remains challenging to radiologists because of the nonspecific nature of conventional abdominal imaging, with a moderate accuracy of approximately $50 \%{ }^{1,2}$ Although detection of malignant FSLs is rare, primary or secondary malignant tumors can seriously damage splenic function. ${ }^{3}$ Histological diagnoses, including splenic biopsy and splenectomy, are rarely accomplished because of severe complications, such as hemorrhage, iatrogenic splenosis and sepsis. $^{3-5}$ Therefore, the investigation of an alternative option for preoperative imaging diagnosis is of vital importance to avoid unnecessary invasive procedures and to conserve the essential immunological function of the spleen. 
With the development of contrast agents, contrastenhanced ultrasound (CEUS) is strongly recommended by the European Federation of Societies for Ultrasound in Medicine and Biology (EFSUMB) as an imaging method for the detection and characterization of focal lesions in the liver, pancreas, and kidney. ${ }^{6-9}$ Studies have shown that spleen-specific uptake and high accumulation of the secondgeneration contrast agent SonoVue enable CEUS to assess in real-time splenic lesions in arterial and parenchymal phases. $^{10,11}$ Previous studies have suggested that CEUS has additional value in the diagnosis of functional asplenia, accessory spleen and vascular splenic pathology, including infarction and trauma. ${ }^{11-16}$ However, the CEUS features of FSLs have not yet been extensively and systematically investigated, and the study of differentiating benign and malignant FSLs by CEUS is also rare. ${ }^{1,17-19}$

In this study, we investigated the imaging features of splenic lesions on conventional ultrasound (B-mode US) and CEUS and further assessed the additional value of CEUS for differentiating malignancy from benignancy.

\section{Patients and Methods}

\section{Patients}

We collected ultrasound reports for the CEUS examination acquired between January 2011 and December 2019 from the database of our department, and we found 1953 patients using the search terms "spleen" or "splenic". Figure 1 shows the flowchart of patient selection. Finally, 123 patients (62 men, 61 women; mean age 50.5 years; age range 19-83 years) with a mean focal splenic lesion size of $3.2 \mathrm{~cm}$ were included in this study. The standard of reference (SOR) confirmed a total of 83 benign lesions ( 48 hemangiomas, 7 hamartomas, 7 hematolymphangiomas, 3 lymphangiomas, 2 sclerosing angiomatoid nodular transformation (SANT), 13 splenic infractions and 3 complex cysts) and 40 malignant lesions (16 lymphomas, 22

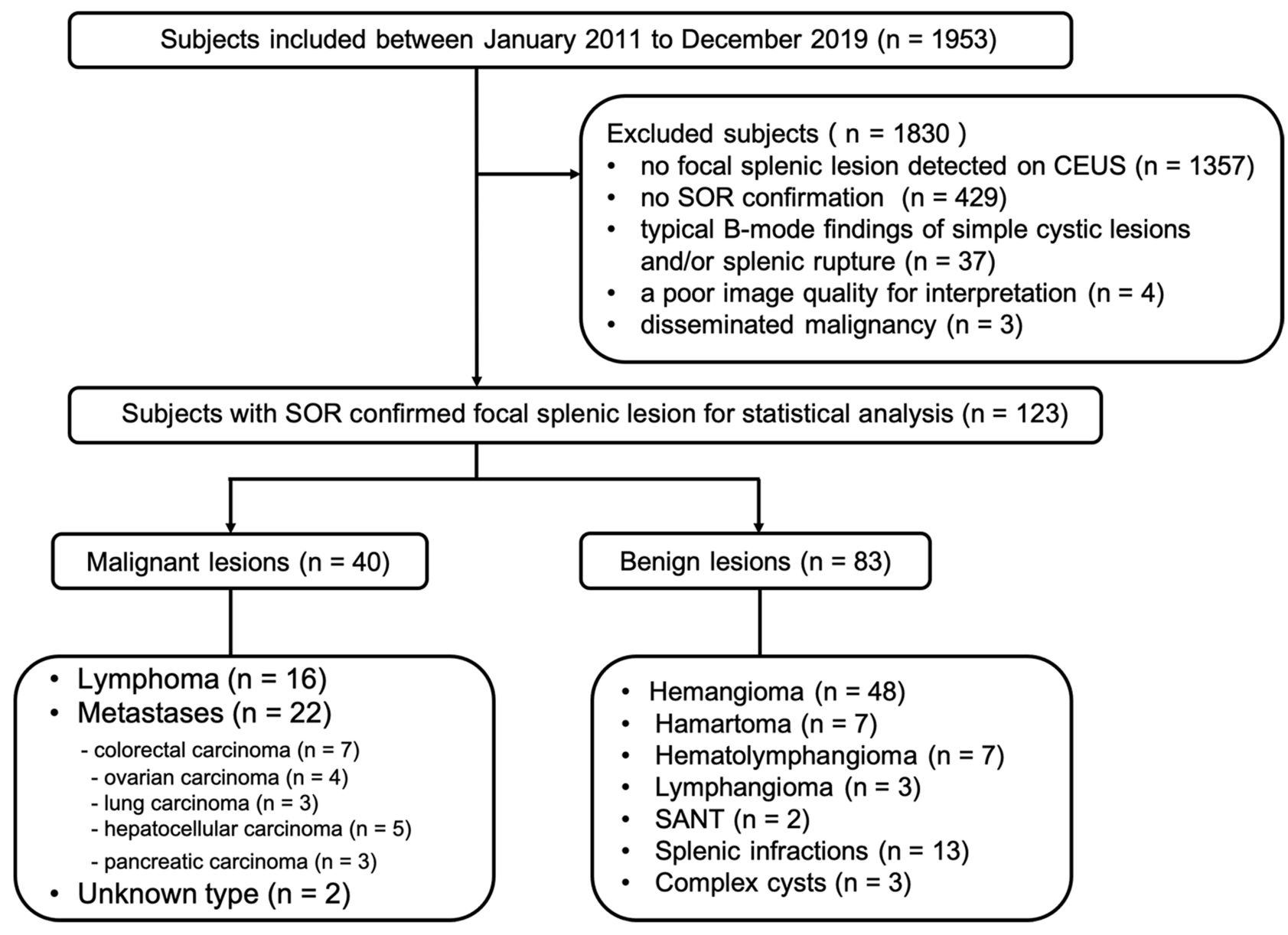

Figure I Flow chart of patient enrollment.

Abbreviations: CEUS, contrast-enhanced ultrasound; SOR, standard of reference; SANT, sclerosing angiomatoid nodular transformation. 
metastases including 7 colorectal carcinomas, 4 ovarian carcinomas, 3 lung carcinomas, 5 hepatocellular carcinomas, 3 pancreatic carcinomas and 2 unknown type). Of the 123 lesions, 37 were confirmed by surgery $(\mathrm{n}=29)$ or biopsy $(n=8)$. The remaining 86 lesions were confirmed by CT $(\mathrm{n}=75)$ and/or MRI $(\mathrm{n}=11)$. Of the 123 patients with focal splenic lesions, 65 patients had a single lesion, and 58 patients had multiple lesions ( $\geq 2$ lesions). In patients with multiple lesions, only the largest lesion was selected for evaluation.

\section{Standard of Reference}

In this study, the most preferred SOR was histopathology by surgery or imaging guided fine-needle biopsy. The alternative method to SOR for the diagnosis of benignancy was based on the typical features of benign splenic lesions without extrasplenic malignancy and changes in lesion size on dynamic enhanced CT or MRI. The alternative method to SOR for the diagnosis of malignancy was to combine representative imaging features of nodular spleen involvement on contrast-enhanced CT and/or MR imaging and/or biopsy-proven malignancy at another extrasplenic site and/or decrease in size after chemotherapy. According to the established CT and MRI criteria, ${ }^{4,20-22}$ dynamic contrast-enhanced $\mathrm{CT}$ or MR images were interpreted by two senior radiologists who had at least 10 years of experience in abdominal CT and MRI.

\section{Ultrasound Examination}

All B-mode US and CEUS examinations were performed by ultrasound doctors with certificates of National Health Commission of the People's Republic China, using an IU22 ultrasound system (Philips Medical Solutions, Mountain View, Calif) with a $\mathrm{C} 5-1 \mathrm{MHz}$ convex or an L9-3 MHz linear probe. A mechanical index of less than 0.1 and pulse inversion harmonic imaging were used for CEUS. Imaging parameters were set constant during the examination, and a dual-screen format with the CEUS image alongside the grayscale image was used to accurately locate the lesion during the procedure. The focal zone was placed below the region of interest, and the selected plane remained unchanged. All patients fasted for at least 8 hours before the examination.

For CEUS, a bolus injection of $2.4 \mathrm{~mL}$ of sulfur hexafluoride filled microbubble contrast agent (SonoVue $^{\mathrm{TM}}$; Bracco, Milan, Italy) was administered via a 20-gauge catheter line placed in the antecubital vein, followed by a 5 -mL flush of $0.9 \%$ sodium chloride solution. The imaging timer was started simultaneously with the completion of the contrast agent injection, and the arteriaphase and parenchymal phase images were obtained 5-30 seconds and 60-300 seconds after injection, respectively. The region of interest, including target lesions and surrounding spleen parenchyma, was imaged continuously for 5 minutes or longer. To minimize motional artifacts, patients were asked to remain still and to try to hold their breath during the examination, particularly during early contrast enhancement. The results of ultrasound examination (images or video recordings) were stored on the hard drive of the ultrasound system and copied to the hard disk of a portable computer for later evaluation.

\section{Imaging Assessment}

The following basic demographic data of patients were collected: sex, age, diagnosis of the underlying tumor disease, and the SOR of splenic lesions.

All B-mode and CEUS images were anonymized and randomly displayed in the sequence (a) B-mode, (b) arterial phase and (c) parenchymal phase of CEUS scans. The images were reviewed in two separate review sessions by two experienced radiologists who had at least 10 years of experience in abdominal ultrasound and 5 years of experience with CEUS. If there were any inconsistencies, a consensus was reached through discussion with the third observer. None of the observers were involved in the scanning, and all were blinded to the clinical, histopathological or other imaging results of each case.

For B-mode US assessment, the following imaging findings of each case were recorded: (a) size of the largest lesion (maximum diameter); (b) echogenicity of lesions (hyperechoic; isoechoic, or hypoechoic); (c) number of lesions (single; multiple); (d) margin (smooth and well defined; almost well defined; or rough and ill-defined); and (e) presence of splenomegaly (maximum width or length $>12 \mathrm{~cm}$ ).

For CEUS, the relative hypoechoic pattern of the lesion compared to the surrounding spleen parenchyma was recorded and classified as follows: (a) no enhancement (no enhancement in the arterial and parenchymal phase); (b) arterial hyperenhancement (partial or entire lesions showing iso-/hyperenhancement in the arterial phase, with slow and incomplete washout in the parenchymal phase); (c) hypoenhancement (although the degree of enhancement in arterial phase was different, the enhancement in parenchymal phase was significantly lower than that in normal spleen); and (d) delayed hyperenhancement 
(hypoenhancement in the arterial phase, with partial or entire iso-/hyperenhancement in the parenchymal phase). In addition, other features on CEUS of each lesion were also recorded and characterized: (a) shape after enhancement (regular, almost regular, irregular, wedge shaped); (b) presence or absence of cystic/necrotic portion; and (c) presence or absence of irregular intralesional vessels.

According to the collected data, two other radiologists independently reviewed B-mode and CEUS sonograms and diagnosed the lesions as (1) definitively benign, (2) probably benign, (3) probably malignant or (4) definitively malignant. The diagnostic efficiency in terms of accuracy, sensitivity, specificity, positive and negative predictive values, and area under the receiver operating characteristic curve (AUC) of B-mode US and CEUS was compared.

\section{Statistical Analysis}

All statistical analyses were performed using commercially available statistical software (SPSS for Mac, version 26.0; SPSS, Chicago, IL). For differentiating benign and malignant splenic lesions, the $X^{2}$ test or Fisher's exact test was used to compare the frequency of qualitative data; Student's $t$-test was used for quantitative analysis and presented as the mean \pm standard deviation. To determine the variables associated with the predictive malignancy of splenic lesions, binary logistic regression analysis with stepwise forward variable selection was conducted using univariate analysis $(P$ values for entry and removal were 0.05 and 0.10 , respectively).

A receiver operating curve analysis was performed to estimate the diagnostic performance and confidence for correct lesion diagnosis. The value of diagnostic performance was calculated in terms of sensitivity, specificity, accuracy, positive predictive value (PPV) and negative predictive value (NPV). Weighted kappa statistics were used to assess the interreader agreement in the diagnostic confidence of the conventional and contrast-enhanced sonograms. The degree of agreement was classified as poor $(\kappa<0.20)$, moderate $(\kappa, 0.20$ to $<0.40)$, fair $(\kappa, 0.40$ to $<0.60$ ), good $(\kappa, 0.60$ to $<0.80$ ), or pretty good ( $\kappa$, $0.80-1.00)$. Statistical significance was indicated by $P$ value less than 0.05 .

\section{Results}

The characteristics of patients and imaging findings between malignant and benign splenic lesions on B-mode and CEUS are given in Table 1. For B-mode US, there were significant differences in lesion echogenicity and
Table I Characteristics of Patients and Imaging Findings

\begin{tabular}{|c|c|c|c|}
\hline \multirow[t]{2}{*}{ Characteristics } & \multirow{2}{*}{$\begin{array}{l}\text { Benignancy } \\
n=83\end{array}$} & \multirow{2}{*}{$\begin{array}{l}\text { Malignancy } \\
n=40\end{array}$} & \multirow[t]{2}{*}{ P-value } \\
\hline & & & \\
\hline Sex & & & 0.654 \\
\hline Male & $43(5 । .8)$ & $19(47.5)$ & \\
\hline Female & $40(48.2)$ & $21(52.5)$ & \\
\hline Age & & & 0.175 \\
\hline Mean & 47.9 & 55.8 & \\
\hline Range & $19-83$ & $27-82$ & \\
\hline Size & & & 0.738 \\
\hline Mean $(\mathrm{cm})$ & 3.2 & 3.2 & \\
\hline Range (cm) & $0.5-20$ & $0.5-10$ & \\
\hline Echogenicity & & & $<0.001$ \\
\hline Hypoechoic & $35(47.2)$ & $35(87.5)$ & \\
\hline Hyperechoic & $40(48.2)$ & $2(5.0)$ & \\
\hline Isoechoic & $8(9.6)$ & $3(7.5)$ & \\
\hline Multiplicity & & & 0.661 \\
\hline Single & $45(54.2)$ & $20(50.0)$ & \\
\hline Multiple & $38(45.8)$ & $20(50.0)$ & \\
\hline Margin & & & 0.243 \\
\hline Well-defined & $24(28.9)$ & $9(22.5)$ & \\
\hline Almost well-defined & $33(39.8)$ & $12(30.0)$ & \\
\hline III defined & $26(31.3)$ & $19(47.5)$ & \\
\hline Splenomegaly & & & 0.012 \\
\hline Yes & $28(33.7)$ & $23(57.5)$ & \\
\hline No & $55(66.3)$ & $17(42.5)$ & \\
\hline Hypoechoic pattern & & & $<0.001$ \\
\hline No enhancement & $17(20.5)$ & $0(0.0)$ & \\
\hline Hypoenhancement & $21(25.3)$ & $39(97.5)$ & \\
\hline Arterial & $32(38.6)$ & I (2.5) & \\
\hline hyperenhancement & & & \\
\hline Delayed & $13(15.7)$ & $0(0.0)$ & \\
\hline hyperenhancement & & & \\
\hline Shape after & & & 0.966 \\
\hline enhancement & & & \\
\hline Regular & $47(59.6)$ & II (27.5) & \\
\hline Almost regular & II (13.3) & $21(52.5)$ & \\
\hline Irregular & $12(14.5)$ & $8(20.0)$ & \\
\hline Wedge shaped & $13(15.7)$ & $0(0.0)$ & \\
\hline Cystic/necrotic portion & & & 0.033 \\
\hline Present & $10(12.0)$ & II (27.5) & \\
\hline Absent & $73(88.0)$ & $29(72.5)$ & \\
\hline Intralesional vessels & & & $<0.001$ \\
\hline Present & $2(16.9)$ & $23(57.5)$ & \\
\hline Absent & 81 (97.6) & $17(42.5)$ & \\
\hline
\end{tabular}

splenomegaly between malignant and benign cases $(P<$ 0.05). Most malignancies appeared hypoechoic (35/40, $87.5 \%$ ), while benign lesions mainly appeared hyperechoic 
(35/83, 48.2\%). Compared with benign lesions, malignant tumors more frequently showed splenomegaly (23/40, $57.5 \%$ ). For CEUS images, malignant splenic lesions more frequently displayed a hypoenhanced pattern (39/ $40,97.5 \%$ ). To be specific, the enhancement of most lesions was substantially lower than that of the spleen in the parenchymal phase followed by rapid and complete washout (Figures 2 and 3). The patterns of CEUS with no enhancement and delayed hyperenhancement only appeared in benign lesions. The hyperenhanced patterns including arterial hyperenhancement and delayed hyperenhancement were mainly observed in benign splenic lesions (Figure 4). All splenic infractions and complex cysts were shown to be completely nonenhanced with a diagnostic accuracy of $100 \%$. The percentage of hypoenhancement pattern was significantly lower in the benign group than in the malignant group $(21 / 83$ vs $39 / 40, P<0.001)$. Furthermore, malignant splenic lesions more commonly showed the presence of cystic or necrotic portions $(P=$ $0.033)$ and intralesional vessels $(P<0.001)$.

Table 2 summarizes the characteristic features predicting malignant focal splenic lesions. Three independent features were identified in the final step of the logistic regression analysis: hypoechoic pattern (odds ratio [OR] $=10.791 ; 95 \%$ confidence interval $[\mathrm{CI}]=2.465,47.239$; $P<0.001)$, hypoenhancement pattern $(\mathrm{OR}=70.026$; $95 \%$ $\mathrm{CI}=13.550,213.420 ; P<0.001)$ and the presence of intralesional vessels $(\mathrm{OR}=29.030 ; 95 \% \mathrm{CI}=2.792$, 301.813; $P=0.005)$. When combining these three features as a predictor for splenic malignant lesions, the sensitivity, specificity, PPV, NPV and accuracy were 22/40 (55.0\%), $83 / 83$ (100.0\%), 22/22 (100.0\%), 83/101 (82.2\%), and $105 / 123(85.4 \%)$, respectively.

Based on the analyses of the above imaging characteristics and previous studies, ${ }^{17-19}$ we proposed the criteria for the prediction of benign and malignant splenic lesions (Table 3). Table 4 summarizes the diagnostic results of other two readers for the 83 benign and 40 malignant splenic lesions based on B-mode US and CEUS criteria. For both readers, the rate of definite diagnosis of benign or malignant splenic lesions for B-mode US was less than $10 \%$, while the rate was significantly increased after reviewing CEUS sonograms (from $10.8 \%$ and $8.4 \%$ to $60.2 \%$ and $61.4 \%$ for benign lesions and from $17.5 \%$ and $12.5 \%$ to $65.0 \%$ and $65.0 \%$ for malignant lesions, respectively; $P<0.05$ ). In addition, the interreader agreement was improved after reviewing CEUS (weighted $\kappa=0.53$ for B-mode versus 0.78 for CEUS).
Table 5 presents the diagnostic efficiency of the two readers for differentiating benign and malignant lesions based on B-mode US and CEUS. Figure 5 shows the individual improvement in diagnostic confidence using receiver operating curve analysis. Compared with B-mode US, CEUS significantly improved the diagnostic performance (overall accuracy $58.5 \%$ and $52.0 \%$ versus $87.0 \%$ and $85.4 \%$, respectively; $P<0.001$ ) and the diagnostic confidence (AUC values 0.622 and 0.533 before versus 0.908 and 0.906 after CEUS for both readers, respectively; $P<0.05$ ) for correct diagnosis of benignancy or malignancy.

\section{Discussion}

With the advent of CEUS and second-generation contrast agents (such as SonoVue ${ }^{\mathrm{TM}}$ ), the diagnostic efficiency of ultrasound in abdominal examination has been significantly improved, especially for most liver lesions, and its sensitivity and specificity are similar to those of $\mathrm{CT}^{23}$ or MRI. ${ }^{24}$ Moreover, emerging data demonstrated that CEUS can be safely applied in pregnant patients with parenchymal lesions and may add pivotal diagnostic value. ${ }^{25,26}$ Supplementary to B-mode US, CEUS can provide more microvascular information in lesions and make the contour and shape of lesions clearer. SonoVue, made of sulfur hexafluoride gas microbubbles, is a pure blood pool contrast agent that is specifically captured by spleen macrophages and does not disperse into the interstitial space. ${ }^{10,27}$ CEUS can be used to differentiate various benign and malignant splenic lesions and to detect traumatic splenic lesions. ${ }^{13,14}$ However, due to the low occurrence of diseases, the number of CEUS studies is scarce, and the diagnostic criteria of splenic lesions are not clearly defined.

In this study, we enrolled 123 patients who underwent B-mode US and CEUS, and the imaging features of each method were compared in splenic lesions to further assess the value of CEUS in splenic lesion diagnosis. Our results indicated that CEUS not only enhances the diagnostic efficiency of detecting splenic benign and malignant lesions but also improves the reliability and repeatability of ultrasound examination. Previous studies reported that most splenic masses are benign and malignant tumors account for only $20-35 \%,{ }^{28,29}$ which is consistent with our results (40/123, 32.5\%; Table 1). Among 83 benign lesions, 13 infarctions and 3 complex cysts presented nonenhancement throughout the CEUS examination. The other 67 lesions displayed various hypoechoic patterns, 

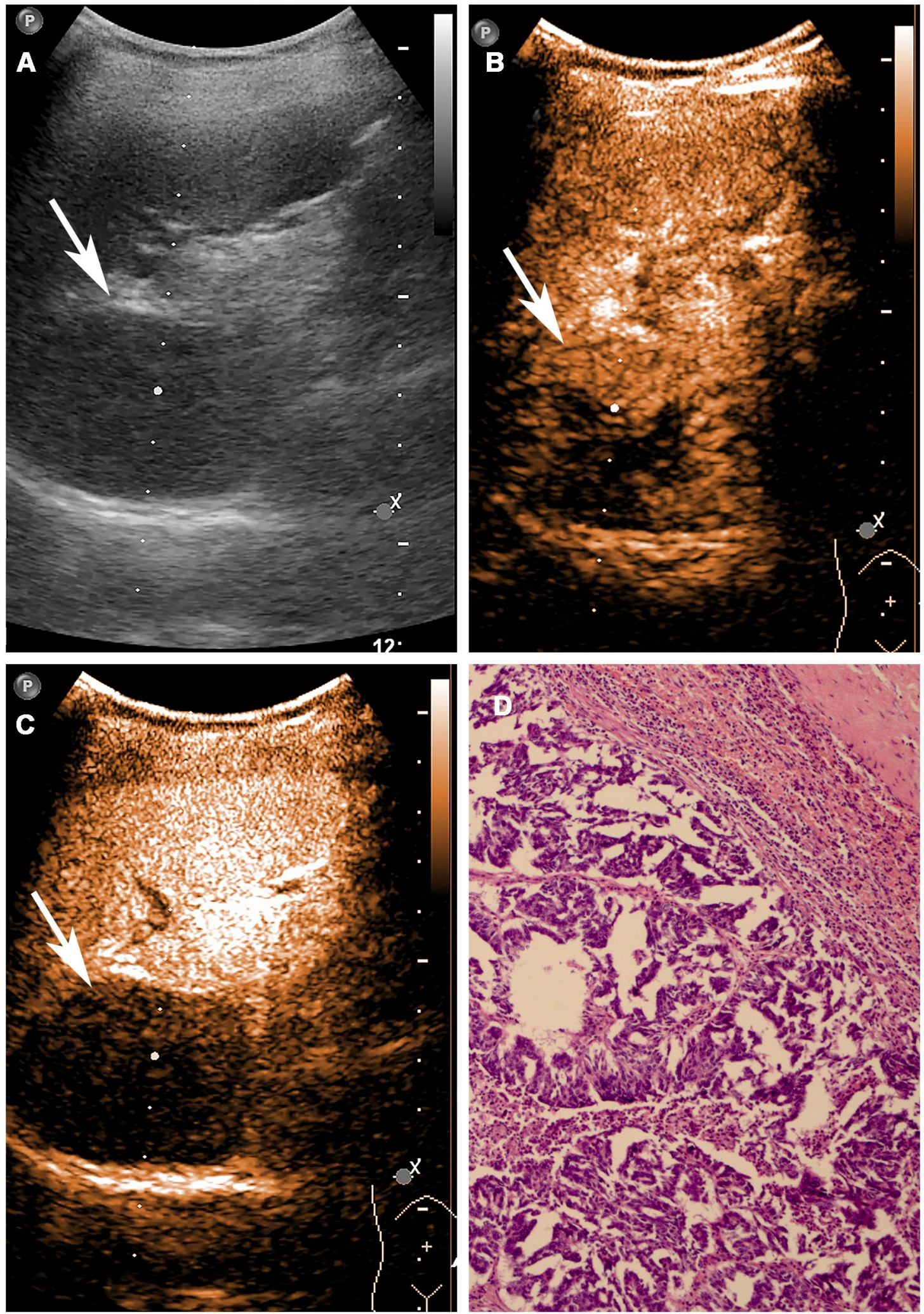

Figure 2 Splenic metastasis in a 53-year-old female patient. (A) B-mode US shows a normal-sized spleen with $4.5 \mathrm{~cm}$ in diameter inhomogeneous hypoechoic lesion (arrow) with almost regular shape. (B) CEUS shows heterogeneously iso-enhancing (arrow) lesion with some small nonenhanced areas (23 seconds after contrast injection). (C) CEUS shows hypoenhanced lesion with dotted aspect (arrow), followed by rapid and complete wash out in parenchymal phase ( 97 seconds after contrast injection). (D) Photograph of corresponding pathologic specimen through hematoxylin-eosin staining (100 magnification). 

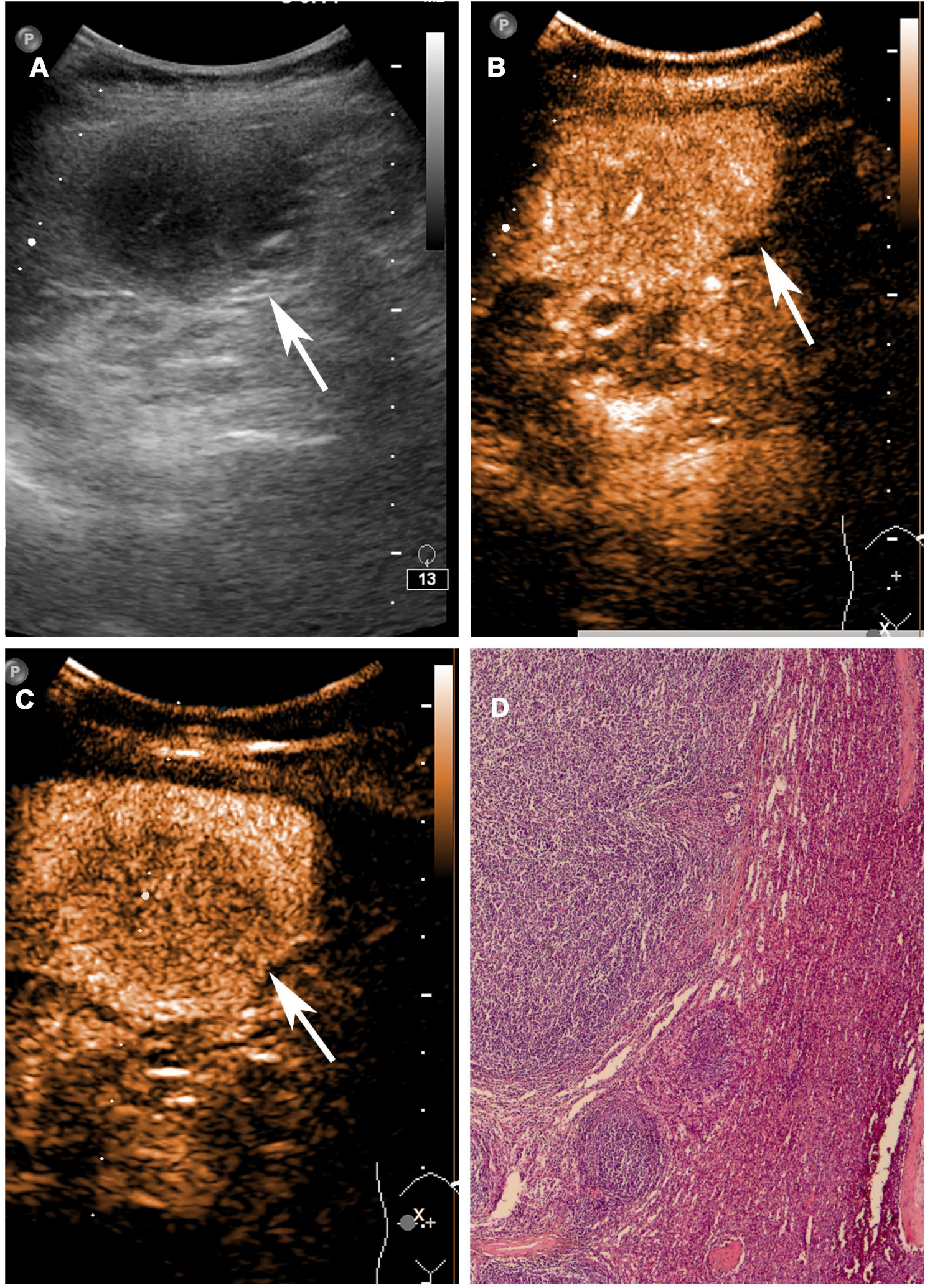

Figure 3 Splenic non-Hodgkin's lymphoma in a 4I-year-old female patient. (A) B-mode US shows a normal-sized spleen with a $4.2 \mathrm{~cm}$ in diameter inhomogeneous hypoechoic lesion (arrow). (B) CEUS shows heterogeneously iso/hyperenhanced lesion (arrow) with irregular vessels in arterial phase (I5 seconds after contrast injection). (C) CEUS shows the enhancement (arrow) in parenchymal phase was lower than that in normal spleen in parenchymal phase ( 3 minutes after contrast injection). (D) Photograph of corresponding pathologic specimen through hematoxylin-eosin staining (40 magnification). 

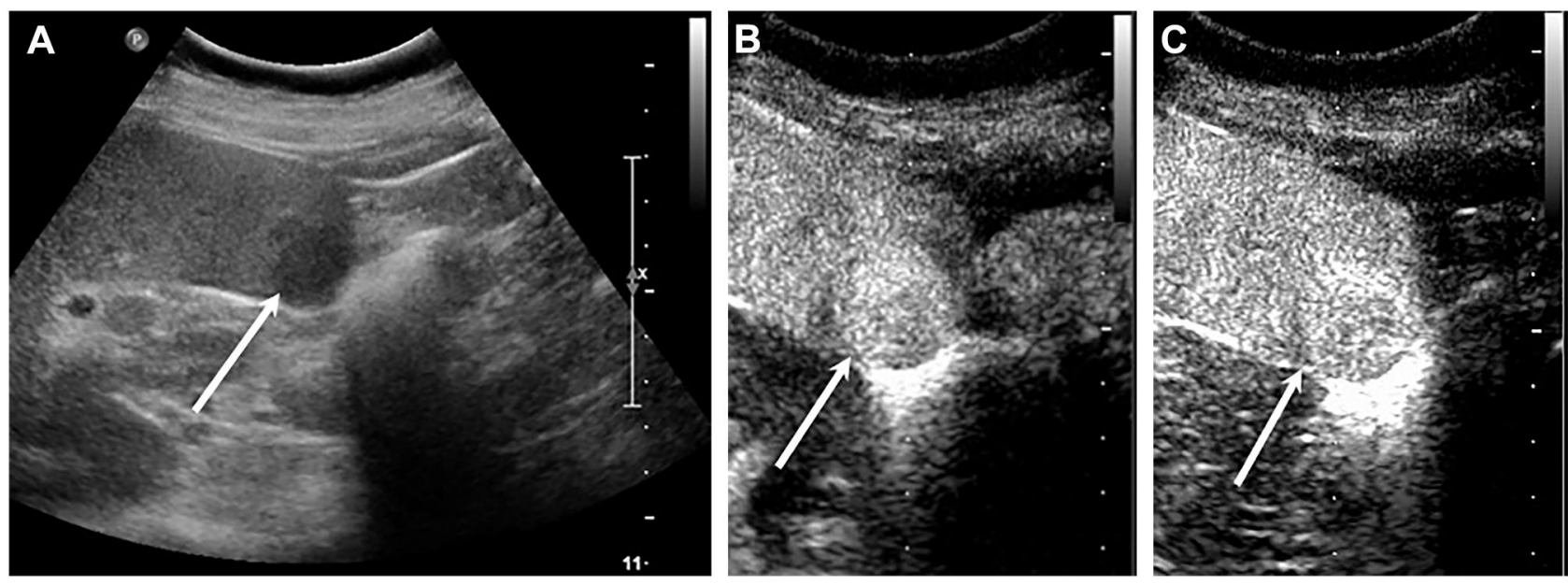

Figure 4 Splenic benign vascular tumor in a 48-year-old female patient. (A) B-mode US shows a normal-sized spleen with a $2.4 \mathrm{~cm}$ in diameter almost well-defined hypoechoic lesion (arrow). (B) CEUS shows hyper-enhancing (arrow) in arterial phase (17 seconds after contrast injection). (C) CEUS shows sustained hyper/iso-enhancing (arrow) in parenchymal phase (I05 seconds after contrast injection).

and most of the lesions were followed by slow and incomplete washout. For malignant lesions, almost all lesions showed hypoenhanced in the parenchymal phase, regardless of the degree of enhancement in the arterial phase, and the contrast washout rate was faster than that of surrounding normal spleen tissues. These findings were in accordance with previous studies that indicated a lower microbubble uptake in malignant splenic lesions than in splenic benign vascular neoplasms such as hemangiomas and/or hamartomas. ${ }^{1,3,15,19,30}$ The absence of microbubble uptake may also allow for differentiating splenic infarcts $^{12,30-32}$ as well as avascular cystic parts from solid splenic tumors. ${ }^{11,30}$

Most splenic lesions are detected incidentally on imaging findings, and the common types of benign lesions are vascular neoplasms, such as hemangiomas and hamartomas. ${ }^{22}$ Several studies ${ }^{15-17,33}$ demonstrated that benign vascular neoplasms typically presented initially iso-/hyperenhancement in the arterial phase followed by slow or incomplete washout. Our results corroborate these findings, showing that most hyperenhanced nodules are benign splenic lesions (32/33). Splenic hemangiomas, arising from the sinusoidal epithelium or cavernous vascular channels, have three typical features, including vascularity, sinusoidal reticuloendothelial system cells (RES), and heterogeneity imaging presentation. ${ }^{34}$ The higher enhancement in the arterial phase is likely due to hypervascular structure with increased arterial supply, ${ }^{22,35,36}$ while no or minimal washout was observed in the parenchymal phase, showing spleen-like retention of microbubbles. ${ }^{10,20}$

Splenic malignant tumors are relatively uncommon, and knowledge of malignancy is crucial for further therapeutic decision making. Due to the lack of sinusoidal spaces and RES cells in malignant lesions, ${ }^{18,19}$ the uptake and retention of microbubbles are significantly lower than those of benign splenic lesions, and the detection rate could be as high as $87 \%{ }^{19}$ Therefore, hypoenhancement in the parenchymal phase of CEUS may be an important feature in the differentiation of malignant tumors. However, our results show that $21 / 83(25.3 \%)$ benign lesions had a similar pattern of hypoenhancement. A few reasons can be considered: hypoenhancement may indicate perfused solid splenic

Table 2 Important Features for Prediction of Malignant Focal Splenic Lesions

\begin{tabular}{|l|c|c|c|c|}
\hline \multicolumn{2}{|l}{} & \multicolumn{3}{c|}{ Multivariable } \\
\cline { 3 - 5 } & OR & $95 \%$ Cl & P-value \\
\hline Echoic pattern & Hypoechoic & 10.791 & $2.465,47.239$ & $<0.001$ \\
\hline Hypoechoic pattern & Hypoenhancement & 70.026 & $13.550,213.420$ & $<0.001$ \\
\hline Intralesional vessels & Presence & 29.030 & $2.792,301.813$ & 0.005 \\
\hline
\end{tabular}

Abbreviations: $\mathrm{Cl}$, confidence interval; $\mathrm{OR}$, odds ratio. 
Table 3 Diagnostic Criteria for Benign and Malignant Splenic Lesions

\begin{tabular}{|c|c|c|c|}
\hline & & Benign Lesions & Malignant Lesions \\
\hline \multirow{3}{*}{$\begin{array}{l}\text { B-mode } \\
\text { US } \\
\text { criteria }\end{array}$} & Echogenicity & 50-70\% hyperechoic & 80-90\% hypoechoic \\
\hline & Echotexture & Homogeneous & Predominantly homogeneous \\
\hline & Margin & Mainly well-defined & $\begin{array}{l}\text { Almost well-defined, sometimes with irregular } \\
\text { delineation }\end{array}$ \\
\hline \multirow[t]{6}{*}{$\begin{array}{l}\text { CEUS } \\
\text { criteria }\end{array}$} & \multirow[t]{4}{*}{ Hypoechoic pattern } & $\begin{array}{l}\text { (a) No enhancement. (no enhancement in the } \\
\text { arterial and parenchymal phase) }\end{array}$ & \multirow{4}{*}{$\begin{array}{l}\text { Mainly hypoenhancement (although the degree } \\
\text { of enhancement in arterial phase was different, } \\
\text { the enhancement in parenchymal phase was } \\
\text { significantly lower than that in normal spleen) }\end{array}$} \\
\hline & & $\begin{array}{l}\text { (b) Arterial hyperenhancement (partial or entire } \\
\text { lesions showing iso-/hyperenhancement in the } \\
\text { arterial phase, with slow and incomplete wash- } \\
\text { out in the parenchymal phase) }\end{array}$ & \\
\hline & & $\begin{array}{l}\text { (c) Hypoenhancement (mainly found in hypere- } \\
\text { choic lesions) }\end{array}$ & \\
\hline & & $\begin{array}{l}\text { (d) Delayed hyperenhancement (hypoenhancement } \\
\text { in the arterial phase, with partial or entire iso-/ } \\
\text { hyperenhancement in the parenchymal phase) }\end{array}$ & \\
\hline & Degree of wash out & $\begin{array}{l}\text { Slow ( }>60 \mathrm{~s} \text { after injection) and incomplete } \\
\text { washout (residual microbubbles uptake }>180 \\
\mathrm{~s} \text { after injection) }\end{array}$ & $\begin{array}{l}\text { Rapid (<60 s after injection) and complete } \\
\text { washout (no residual microbubble }>180 \mathrm{~s} \text { after } \\
\text { injection) }\end{array}$ \\
\hline & Other characteristics & $\begin{array}{l}\text { Sometimes wedge-shaped or round defect may be } \\
\text { seen }\end{array}$ & $\begin{array}{l}\text { Sometimes irregular intralesional vessels and/or } \\
\text { non-perfused cystic areas may be seen }\end{array}$ \\
\hline
\end{tabular}

lesions of tissue devoid of sinusoids ${ }^{19}$ and lower microbubble uptake in hypoechoic splenic hemangiomas. ${ }^{1,10,16,17}$ Taibbi et $\mathrm{al}^{37}$ reported that 8 of 27 hemangiomas showed some degree of enhancement on CEUS but remained substantially lower than that of the surrounding splenic tissue. Stang et al ${ }^{19}$ reported that the most apparent overlap between benign and malignant splenic lesions was seen with nodular sarcoidosis and lymphoma, both of which were

Table 4 Diagnostic Results of 123 Splenic Lesions on B-Mode Ultrasound and Contrast-Enhanced Ultrasound n (\%)

\begin{tabular}{|c|c|c|c|c|c|}
\hline & & \multicolumn{2}{|c|}{ Reader I } & \multicolumn{2}{|c|}{ Reader2 } \\
\hline & & Benign Lesions $(n=83)$ & Malignant Lesions $(n=40)$ & Benign Lesions $(n=83)$ & Malignant Lesions $(n=40)$ \\
\hline \multicolumn{6}{|c|}{ B-mode ultrasound } \\
\hline \multirow[t]{2}{*}{ Benign } & Definitely & $9(10.8)$ & $\mathrm{I}(2.5)$ & $7(8.4)$ & I (2.5) \\
\hline & Probably & $37(44.6)$ & $13(32.5)$ & $34(41.0)$ & $16(40.0)$ \\
\hline \multirow[t]{2}{*}{ Malignant } & Probably & $29(34.9)$ & $19(47.5)$ & $29(34.9)$ & $18(45.0)$ \\
\hline & Definitely & $8(9.6)$ & $7(17.5)$ & $13(15.7)$ & $5(12.5)$ \\
\hline \multicolumn{6}{|c|}{ Contrast-enhanced ultrasound } \\
\hline \multirow[t]{2}{*}{ Benign } & Definitely & $50(60.2)$ & I (2.5) & $5 I(6 I .4)$ & I (2.5) \\
\hline & Probably & $20(24.1)$ & $2(5.0)$ & $16(19.3)$ & I (2.5) \\
\hline \multirow[t]{2}{*}{ Malignant } & Probably & $6(7.2)$ & II (27.5) & $9(10.8)$ & $12(30.0)$ \\
\hline & Definitely & $7(8.4)$ & $26(65.0)$ & $7(8.4)$ & $26(65.0)$ \\
\hline
\end{tabular}


Table 5 Diagnostic Efficiency of B-Mode Ultrasound and ContrastEnhanced Ultrasound Between Benign and Malignant Splenic Lesions

\begin{tabular}{|l|l|c|c|c|}
\hline $\begin{array}{l}\text { Features } \\
\text { of } \\
\text { Lesions }\end{array}$ & Readers & $\begin{array}{c}\text { B-mode } \\
\text { Ultrasound }\end{array}$ & $\begin{array}{c}\text { Contrast- } \\
\text { Enhanced } \\
\text { Ultrasound }\end{array}$ & P value \\
\hline Accuracy & ReaderI & $58.5 \%$ & $87.0 \%$ & $<0.001$ \\
& Reader2 & $52.0 \%$ & $85.4 \%$ & $<0.001$ \\
\hline Sensitivity & Reader I & $65.0 \%$ & $92.5 \%$ & 0.003 \\
& Reader2 & $57.5 \%$ & $95.0 \%$ & $<0.001$ \\
\hline Specificity & Reader I & $55.4 \%$ & $84.3 \%$ & $<0.001$ \\
& Reader2 & $49.4 \%$ & $80.7 \%$ & $<0.001$ \\
\hline PPV & ReaderI & $41.3 \%$ & $74.0 \%$ & 0.001 \\
& Reader2 & $35.4 \%$ & $70.4 \%$ & 0.196 \\
\hline NPV & Reader I & $76.7 \%$ & $95.9 \%$ & 0.001 \\
& Reader2 & $70.7 \%$ & $97.1 \%$ & 0.166 \\
\hline
\end{tabular}

Abbreviations: PPV, positive predictive value; NPV, negative predictive value.

characterized by multiple progressive hypo-enhanced lesions up to $3 \mathrm{~cm}$ in diameter. In our study, we found that most of the enrolled hyperechoic benign vascular neoplasms and SANTs showed this pattern. While most malignant lesions were hypoenhanced in the parenchymal phase, the lesions manifesting with this pattern might not necessarily be malignant. Therefore, hypoenhancement alone is not sufficient to determine the malignancy, and other imaging features of CEUS should be considered, such as rapid and complete washout in the parenchymal phase, irregular intralesional vessels, and dotted aspects. These features have also been observed by other research groups. ${ }^{11,19,38}$ Therefore, in order to better distinguish between benign and malignant splenic lesions, we proposed prediction criteria based on the characteristics of B-ultrasound and contrast-enhanced ultrasound (Table 3). Our results indicated that these criteria were helpful for the differential diagnosis of benign and malignant spleen lesions in this study.

Our study has several limitations. Firstly, not all splenic lesions were confirmed by histopathological examinations. For ethical reasons, biopsies were not systematically performed in patients with lesions that were evaluated as benign based on available imaging data in combination with clinical information. Secondly, compared with benign splenic lesions, the number of malignant splenic lesions was substantially insufficient. Finally, this study only focused on ultrasound imaging modality, while the application of fusion imaging (CEUS plus CT/MRI) may help to elucidate indeterminate parenchymal lesions and further improve the accuracy of diagnosis. ${ }^{39-41}$ Moreover, fusion imaging could be applicated in numerous fields, particularly in the characterization of parenchymal lesions as well as during interventions. ${ }^{39}$ Ewertsen et $\mathrm{al}^{42}$ reported that fusion imaging could help to characterize unclear lesions and clarify the benign or malignant status of a greater number of lesions. The application of
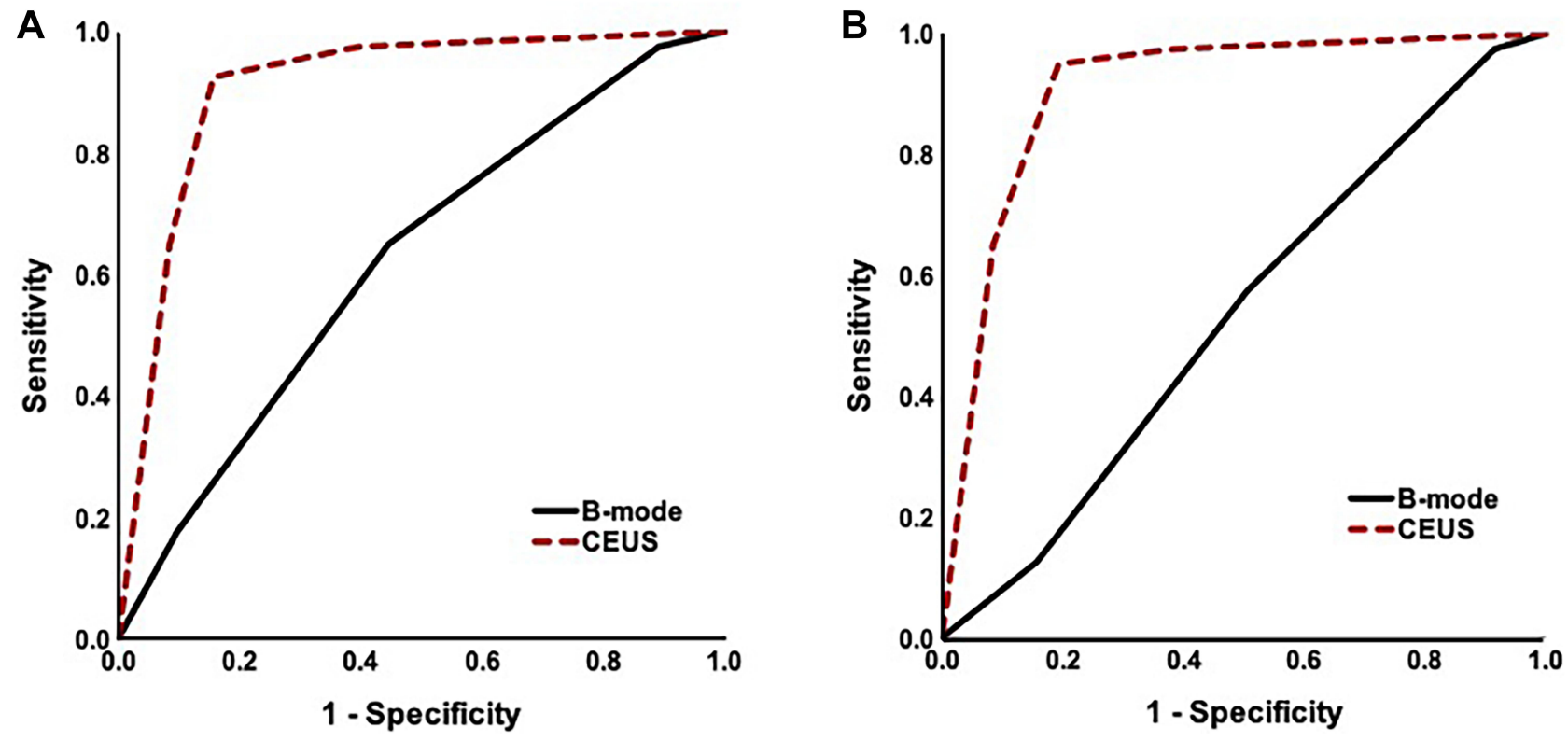

Figure 5 Receiver operating characteristic curves for differentiating benign and malignant splenic lesions after review of B-mode (continuous line) and CEUS (dotted line) sonograms. For reader I (A), the diagnostic confidence increased from 0.622 with B-mode US to 0.908 with CEUS and for reader 2 (B), the diagnostic confidence increased from 0.533 with B-mode to 0.906 with CEUS. The improvement in diagnostic confidence after review of CEUS was statistically significant ( $p<0.00 \mathrm{I})$ for both readers. 
fusion imaging in splenic lesions will be investigated in our further studies.

\section{Conclusion}

In conclusion, CEUS may provide more useful information than conventional ultrasound and significantly improve the diagnostic efficiency for distinguishing malignant from benign splenic lesions. Further optimizing the diagnostic criteria will make CEUS an effective alternative to $\mathrm{CT}$ or MRI and reduce unnecessary histological evaluation in clinical scenarios.

\section{Ethics}

This is a single-center study and was approved by West China Hospital of Sichuan University Biomedical Research Ethics Committee. Written informed consent was waived because of the retrospective nature of clinical and imaging data collection. The study was conducted in accordance with the Declaration of Helsinki and the privacy of the participants data were protected.

\section{Acknowledgments}

We greatly appreciate the financial supports by the 1.3 .5 project for disciplines of excellence, West China Hospital, Sichuan University (ZYJC18008), National Science Foundation of China (NSFC) (81701797), Beijing Natural Science Foundation (7192200), Science and Technology Innovation Talent of Sichuan (20CXRC0065), Science and Technology Project of Chengdu (2019-YF05-00376-SN), Science and Technology Project of the Health Planning Committee of Sichuan (20PJ011).

\section{Disclosure}

The authors report that they have no conflicts of interest related to this work.

\section{References}

1. Yu X, Yu J, Liang P, Liu F. Real-time contrast-enhanced ultrasound in diagnosing of focal spleen lesions. Eur J Radiol. 2012;81(3):430-436. doi:10.1016/j.ejrad.2010.12.052

2. Guibaud L. Sonography of the pediatric abdomen: pancreas and spleen. J Radiol. 2001;82(6 Pt 2):755-761; discussion 762-753.

3. Vancauwenberghe T, Snoeckx A, Vanbeckevoort D, Dymarkowski S, Vanhoenacker FM. Imaging of the spleen: what the clinician needs to know. Singapore Med J. 2015;56(3):133-144.

4. Jang S, Kim JH, Hur BY, et al. Role of CT in differentiating malignant focal splenic lesions. Korean $J$ Radiol. 2018;19(5):930-937. doi:10.3348/kjr.2018.19.5.930
5. Silver DS, Pointer DT Jr, Slakey DP. Solid tumors of the spleen: evaluation and management. $J$ Am Coll Surg. 2017;224 (6):1104-1111. doi:10.1016/j.jamcollsurg.2016.12.043

6. Dietrich CF, Nolsøe CP, Barr RG, et al. Guidelines and good clinical practice recommendations for Contrast Enhanced Ultrasound (CEUS) in the liver - update 2020 - WFUMB in cooperation with EFSUMB, AFSUMB, AIUM, and FLAUS. Ultraschall Med. 2020;41 (5):562-585.

7. Dietrich CF, Cui XW, Boozari B, Hocke M, Ignee A. Contrast-enhanced ultrasound (CEUS) in the diagnostic algorithm of hepatocellular and cholangiocellular carcinoma, comments on the AASLD guidelines. Ultraschall Med. 2012;33(Suppl 1):S57-S66. doi:10.1055/s-0032-1312903

8. Barreiros AP, Piscaglia F, Dietrich CF. Contrast enhanced ultrasound for the diagnosis of hepatocellular carcinoma (HCC): comments on AASLD guidelines. $J$ Hepatol. 2012;57(4):930-932. doi:10.1016/j. jhep.2012.04.018

9. Seitz K, Bernatik T, Strobel D, et al. Contrast-enhanced ultrasound (CEUS) for the characterization of focal liver lesions in clinical practice (DEGUM Multicenter Trial): CEUS vs. MRI-a prospective comparison in 269 patients. Ultraschall Med. 2010;31(5):492-499. doi:10.1055/s-0029-1245591

10. Lim AK, Patel N, Eckersley RJ, Taylor-Robinson SD, Cosgrove DO, Blomley MJ. Evidence for spleen-specific uptake of a microbubble contrast agent: a quantitative study in healthy volunteers. Radiology. 2004;231(3):785-788. doi:10.1148/radiol.2313030544

11. Gorg C. The forgotten organ: contrast enhanced sonography of the spleen. Eur J Radiol. 2007;64(2):189-201. doi:10.1016/j.ejrad.2007.06.036

12. Catalano O, Lobianco R, Sandomenico F, Siani A. Splenic trauma: evaluation with contrast-specific sonography and a second-generation contrast medium: preliminary experience. $J$ Ultrasound Med. 2003;22(5):467-477. doi:10.7863/jum.2003.22.5.467

13. Tang J, Zhang HQ, Lu FQ, Li WX, Luo YK, Li TS. [Role of contrast-enhanced ultrasound in management of splenic trauma]. Zhongguo Yi Xие Ke Xие Yuan Xие Baо. 2008;30(1):27-30. Chinese. PMID: 18361048.

14. Brillantino A, Iacobellis F, Robustelli U, et al. Non operative management of blunt splenic trauma: a prospective evaluation of a standardized treatment protocol. Eur J Trauma Emerg Surg. 2016;42(5):593-598. doi:10.1007/s00068-015-0575-z

15. Gorg C, Graef C, Bert T. Contrast-enhanced sonography for differential diagnosis of an inhomogeneous spleen of unknown cause in patients with pain in the left upper quadrant. $J$ Ultrasound Med. 2006;25(6):729-734. doi:10.7863/jum.2006.25.6.729

16. Gorg C, Bert T. Contrast enhanced sonography of focal splenic lesions with a second-generation contrast agent. Ultraschall Med. 2005;26(6):470-477. doi:10.1055/s-2005-858904

17. Stang A, Keles H, Hentschke S, et al. Incidentally detected splenic lesions in ultrasound: does contrast-enhanced ultrasonography improve the differentiation of benign hemangioma/hamartoma from malignant lesions? Ultraschall Der Medizin. 2011;32(06):582-592. doi:10.1055/s-0031-1282034

18. von Herbay A, Barreiros AP, Ignee A, et al. Contrast-enhanced ultrasonography with SonoVue: differentiation between benign and malignant lesions of the spleen. J Ultrasound Med. 2009;28 (4):421-434. doi:10.7863/jum.2009.28.4.421

19. Stang A, Keles H, Hentschke S, et al. Differentiation of benign from malignant focal splenic lesions using sulfur hexafluoride-filled microbubble contrast-enhanced pulse-inversion sonography. AJR Am J Roentgenol. 2009;193(3):709-721. doi:10.2214/AJR.07.3988

20. Robertson F, Leander P, Ekberg O. Radiology of the spleen. Eur Radiol. 2001;11(1):80-95. doi:10.1007/s003300000528

21. Jang KM, Kim SH, Hwang J, et al. Differentiation of malignant from benign focal splenic lesions: added value of diffusion-weighted MRI. AJR Am J Roentgenol. 2014;203(4):803-812. doi:10.2214/ AJR.13.11914 
22. Abbott RM, Levy AD, Aguilera NS, Gorospe L, Thompson WM. From the archives of the AFIP: primary vascular neoplasms of the spleen: radiologic-pathologic correlation. Radiographics. 2004;24 (4):1137-1163. doi:10.1148/rg.244045006

23. Seitz K, Strobel D, Bernatik T, et al. Contrast-Enhanced Ultrasound (CEUS) for the characterization of focal liver lesions - prospective comparison in clinical practice: CEUS vs. CT (DEGUM multicenter trial). Parts of this manuscript were presented at the Ultrasound Dreilandertreffen 2008, Davos. Ultraschall Med. 2009;30 (4):383-389. doi:10.1055/s-0028-1109673

24. Beyer LP, Wassermann F, Pregler B, et al. Characterization of focal liver lesions using CEUS and MRI with liver-specific contrast media: experience of a single radiologic center. Ultraschall Med. 2017;38 (6):619-625. doi:10.1055/s-0043-105264

25. Schwarze V, Marschner C, Negrão de Figueiredo G, Rübenthaler J, Clevert DA. Single-center study: evaluating the diagnostic performance and safety of contrast-enhanced ultrasound (CEUS) in pregnant women to assess hepatic lesions. Ultraschall Med. 2020;41 (1):29-35. doi:10.1055/a-0973-8517

26. Geyer T, Rübenthaler J, Froelich MF, et al. Contrast-enhanced ultrasound for assessing abdominal conditions in pregnancy. Medicina. 2020;56:12.

27. Wilson SR, Burns PN. Microbubble-enhanced US in body imaging: what role? Radiology. 2010;257(1):24-39. doi:10.1148/ radiol.10091210

28. Dhyani M, Anupindi SA, Ayyala R, Hahn PF, Gee MS. Defining an imaging algorithm for noncystic splenic lesions identified in young patients. AJR Am J Roentgenol. 2013;201(6):W893-W899. doi:10.2214/AJR.12.10105

29. Cao F, Qian W, Ma Y, Wu Y, Zhong J. Contrast-enhanced imaging features and differentiation of benign and malignant focal splenic lesions. Clin Imaging. 2018;49:58-64. doi:10.1016/j. clinimag.2017.10.020

30. Catalano O, Sandomenico F, Matarazzo I, Siani A. Contrastenhanced sonography of the spleen. AJR Am J Roentgenol. 2005;184(4):1150-1156. doi:10.2214/ajr.184.4.01841150

31. Seeger M, Folsch UR. [Diagnosis of acute splenic infarction by echo-signal-enhanced ultrasound]. Dtsch Med Wochenschr. 2004;129(16):876-879. German. doi:10.1055/s-2004-823033

32. Menozzi G, Maccabruni V, Gabbi E, Magnani G, Garlassi E. Evaluation with contrast ultrasound of the prevalence of splenic infarction in left-sided infective endocarditis. J Ultrasound. 2015;18 (3):223-227. doi:10.1007/s40477-014-0131-4
33. Kamaya A, Weinstein S, Desser TS. Multiple lesions of the spleen: differential diagnosis of cystic and solid lesions. Semin Ultrasound CT MR. 2006;27(5):389-403. doi:10.1053/j.sult.2006.06.004

34. Sugihara T, Koda M, Kato J, Sakamoto T, Horie Y, Murawaki Y. Contrast-enhanced sonography with Sonazoid as a new diagnostic tool for splenic hamartoma: a single case report. $J$ med ultrason. 2016;43(1):113-118. doi:10.1007/s10396-015-0660-2

35. Ros PR, Moser RP Jr, Dachman AH, Murari PJ, Olmsted WW. Hemangioma of the spleen: radiologic-pathologic correlation in ten cases. Radiology. 1987;162(1 Pt 1):73-77. doi:10.1148/ radiology.162.1.3538155

36. Chun YS, Robu VG. Spectrum of primary vascular neoplasms of the spleen. J Clin Oncol. 2011;29(5):e116-e117. doi:10.1200/ JCO.2010.31.7230

37. Taibbi A, Bartolotta TV, Matranga D, Midiri M, Lagalla R. Splenic hemangiomas: contrast-enhanced sonographic findings. J Ultrasound Med. 2012;31(4):543-553. doi:10.7863/jum.2012.31.4.543

38. Tafuto S, Catalano O, Barba G, et al. Real-time contrast-enhanced specific ultrasound in staging and follow-up of splenic lymphomas. Front Biosci. 2006;11:2224-2229. doi:10.2741/1965

39. European Society of Radiology (ESR). Abdominal applications of ultrasound fusion imaging technique: liver, kidney, and pancreas. Insights Imaging. 2019;10(1):6. doi:10.1186/s13244-019-0692-z

40. Xu E, Long Y, Li K, et al. Comparison of CT/MRI-CEUS and US-CEUS fusion imaging techniques in the assessment of the thermal ablation of liver tumors. Int $J$ Hyperthermia. 2019;35 (1):159-167. doi:10.1080/02656736.2018.1487591

41. Kloth C, Kratzer W, Schmidberger J, Beer M, Clevert DA, Graeter T. Ultrasound 2020 - diagnostics \& therapy: on the way to multimodal ultrasound: contrast-enhanced ultrasound (CEUS), microvascular doppler techniques, fusion imaging, sonoelastography, interventional sonography. Rofo. 2021;193(1):23-32.

42. Ewertsen C, Henriksen BM, Torp-Pedersen S, Bachmann Nielsen M. Characterization by biopsy or CEUS of liver lesions guided by image fusion between ultrasonography and CT, PET/CT or MRI. Ultraschall Med. 2011;32(2):191-197. doi:10.1055/s-0029-1245921

\section{Publish your work in this journal}

Cancer Management and Research is an international, peer-reviewed open access journal focusing on cancer research and the optimal use of preventative and integrated treatment interventions to achieve improved outcomes, enhanced survival and quality of life for the cancer patient.
The manuscript management system is completely online and includes a very quick and fair peer-review system, which is all easy to use. Visit http://www.dovepress.com/testimonials.php to read real quotes from published authors. 